\title{
Targeting the Radiation-Induced ARv7-Mediated circNHS/miR-512-5p/XRCC5 Signaling With Quercetin Signaling Increases Prostate Cancer Radiosensitivity
}

\section{Dong Chen}

Chinese Academy of Medical Sciences Cancer Institute and Hospital: Cancer Hospital Chinese Academy of Medical Sciences

Fu-Ju Chou

University of Rochester Medical Center

\section{Yuhchyau Chen}

University of Rochester Medical Center

Hao Tian

Tianjin Medical University

\section{Yaqin Wang}

Fuwai Hospital State Key Laboratory of Cardiovascular Disease

\section{Yuanjie Niu}

Tianjin Medical University

\section{Chiping Huang}

China Medical University

\section{Bosen You}

University of Rochester Medical Center

\section{Shuyuan Yeh}

University of Rochester Medical Center

Nianzeng Xing

Chinese Academy of Medical Sciences Cancer Institute and Hospital: Cancer Hospital Chinese Academy of Medical Sciences

Chawnshang Chang ( $\square$ chang@urmc.rochester.edu )

University of Rochester Medical Center

\section{Research}

Keywords: ARv7, circNHS, miR-512-5p, XRCC5, Quercetin, prostate cancer, radiosensitivity

Posted Date: July 7th, 2021 
DOl: https://doi.org/10.21203/rs.3.rs-659472/v1

License: (c) (1) This work is licensed under a Creative Commons Attribution 4.0 International License. Read Full License 


\section{Abstract}

\section{Background}

Radiation therapy (RT) with androgen deprivation therapy (ADT) is an effective therapy to suppress the locally advanced prostate cancer (PCa). However, we unexpected found that RT could also inducing the androgen receptor splice variant 7 (ARv7) expression to decrease the radiosensitivity.

\section{Methods}

The study was designed to target ARv7 expression with Quercetin or ARv7-shRNA led to enhancing increase the radiation sensitivity to better suppress the PCa that involved the modulating the circNHS/miR-512-5p/XRCC5 signaling.

Results

Mechanism studies revealed that RT-induced ARv7 may function via altering the circNHS/miR-512$5 p /$ XRCC5 signaling to decrease the radiosensitivity. Results from preclinical studies using multiple in vitro cell lines and in vivo mouse models concluded that combining RT with small molecule of Quercetin to target full-length AR and ARv7 could lead to better efficacy to suppress PCa progression.

\section{Conclusion}

Together, these results suggest that ARv7 may play key roles to alter the PCa radiosensitivity, and targeting this newly identified ARv7 mediated circNHS/miR-512-5p/XRCC5 signaling with Quercetin may help us to develop a novel RT to better suppress the progression of PCa.

\section{Background}

Prostate cancer ( $\mathrm{PCa}$ ) is the second leading cause of cancer deaths and the most common cancer in men in the United States (1) and its morbidity is also rapidly rising in Asian (2). Radiation therapy (RT) including external beam radiotherapy (EBRT) and brachytherapy is one of the standard treatment modalities for managing primary or locally advanced disease (3). However, there are still $20-25 \%$ of PCa patients with noninvasive disease (stage T1-T2) relapse after high doses of radiation in less than 5 years (1). Clinically, the combination of RT and androgen deprivation therapy (ADT) is superior to RT alone for treatment of patients with localized, intermediate-risk and high-risk PCa, since ADT may abrogate the androgens/androgen receptor (AR)-induced the DNA damage repair (DDR) system so that RT can be more efficiently to suppress $\mathrm{PCa}(4,5)$.

Despite use of combined ADT and RT, biochemical recurrence was up to $50 \%$ of high metastatic risk PCa patients (6); One possible mechanism proposed to explain this was that ADT treatment could induce the expression of AR variants (ARVs) especially AR variants 7 (ARv7) in PCa (7-9) and ARVs could mediate DDR following RT(10). Therefore, it is necessary to inhibit both full-length AR and ARv7 signal pathway to 
effectively improve PCa radiosensitivity. Moreover, there is no study about the effect of ionizing radiation ARVs especially ARv7 expression in PCa.

Quercetin (Que, 3,3',4',5,7-pentahydroxyflavone) is a bioactive plant-derived flavonoid, abundant in fruits and vegetables, that has been used as nutrition supplement in several countries (11). Daily human intake of quercetin ranges from 10 to $100 \mathrm{mg}$ depending on different dietary habits, and it can reach 500-1,000 $\mathrm{mg}$ if selected highly purified extracts are used (12). Recent studies also indicated that Que might have anti-cancer property that could suppress tumor cell growth in multiple in vitro cell lines and in vivo mouse models in many cancers (13-15). Its linkage to the ARVs, especially to the IR-induced ARv7 during RT, however, remains unclear.

Circular RNAs (circRNAs) are highly conserved and stable covalently closed RNA transcripts generated by back-splicing of a single pre-mRNA with gene-regulatory potential $(16,17)$. Emerging evidence shows that circRNAs are closely related to human diseases, especially cancers, and may act as better biomarkers due to their abundance and stability $(18,19)$. circRNAs have also been linked to the radiosensitivity of several cancer types $(20,21)$ and we previously found circZEB1 could decrease radiosensitivity by mediating miR-141-3p/ZEB1 signaling pathway in PCa cells (22).

Here we found that IR may function via inducing the ARv7 expression to decrease the subsequent RT efficacy, and adding Que may then suppress the IR-induced both full-length AR and ARv7 to better increase the PCa radiosensitivity

\section{Methods}

2.1. Cell lines and cell culture. C4-2, $22 \mathrm{Rv} 1$ and $\mathrm{VCaP}$ cell lines were obtained from American Type Culture Collection (ATCC) and maintained in RPMI 1640 medium containing 10\% fetal bovine serum (FBS), antibiotics (100 unit/ml penicillin, $100 \mu \mathrm{g} / \mathrm{ml}$ streptomycin), and $2 \mathrm{mM}$ glutamine (Invitrogen) in a humidified $5 \% \mathrm{CO} 2$ environment at $37^{\circ} \mathrm{C}$. All cell lines were authenticated as mycoplasma and bacteria free following ATCC instructions during the three months prior to experiments.

2.2. Plasmids and lentivirus. The pLKO.1-shAR, pLKO.1-shARv7, pLKO.1-circNHS, pLKO.1-XRCC5, pWPIARv7, pWPI-circNHS and pWPI-XRCC5 plasmids, the psPAX2 packaging plasmid, and pMD2G envelope plasmid (lentivirus:packaging:envelope, 2:1:1) were cotransfected into 293T cells using the standard calcium chloride transfection method for 48 hours to obtain the lentiviral supernatant. The lentiviral supernatants were collected and concentrated by density gradient centrifugation for immediate use or frozen at $-80^{\circ} \mathrm{C}$ for later use.

2.3. Clonogenic survival assays. Cells were plated in $60-\mathrm{mm}$ plates at various densities, depending on the IR dosage, and allowed to attach overnight. Cells were treated with media and metformin or vehicle for 48 hours. Then, the cells were irradiated, and drug treatments were removed as soon as possible after IR, after which the cells were incubated for 14 days. Survival curves for each experiment were constructed by plotting the mean surviving fractions semilogarithmically as a function of the IR dose. The data were 
analyzed, and survival curves were plotted following a multitarget/single-hit model $\left(\mathrm{SF}=1-\left[1-\mathrm{e}^{-\mathrm{D} / \mathrm{D} 0}\right]^{\mathrm{N}}\right)$ to plot the surviving fraction curve using GraphPad Prism 7.0 software, where SF is the surviving fraction and $D$ is the radiation dose.

\subsection{Quantitative real-time PCR. Total RNA was isolated using TRIzol ${ }^{\circledR}$ Reagent (\#15596026}

ThermoFisher Scientific) according to the manufacturer's instructions. Total RNA was reverse transcribed into cDNA using the iScript ${ }^{\text {TM }}$ cDNA Synthesis Kit (\# 1708891, Bio-Rad). The primer sequences are listed in Table S1. qRT-PCR was performed using a Bio-Rad iQ5 real-time thermal cycler and iQ ${ }^{\text {TM }}$ SYBR® Green Supermix (\#1708880, Bio-Rad). Relative mRNA expression levels were normalized against GAPDH levels (as an internal control) and determined by the $2^{-\Delta \Delta C t}$ method.

2.5. Western blot assays. For western blot analyses, protein extracts of each sample ( $50 \mu \mathrm{g} / \mathrm{lane})$ were electrophoretically separated and transferred onto PVDF membranes that were incubated with antibodies against ARv7 (\#ab198394, abcam, USA), AR (\#sc-816, Santa Cruz, USA), Y-H2AX (\#05-636 clone JBW301, EMD Millipore), XRCC5 (\#sc-5309, Santa Cruz, USA), SPRTN (\#PA5-110424, thermofisher, USA) and GAPDH (\#sc-166, Santa Cruz, USA), followed by horseradish peroxidase-conjugated secondary antibody. Protein-antibody complexes were detected by SuperSignal ${ }^{\mathrm{Tm}}$ West Femto Maximum Sensitivity Substrate (\#34095, ThermoFisher Scientific) using the Bio-Rad imaging system.

2.6. Neutral comet assay. Double strand breaks (DSBs) were assessed by a single-cell gel electrophoretic comet assay kits (Trevigen) under neutral conditions according to the manufacturer's protocol. Briefly, cells were harvested at the indicated times after a single dose of $6 \mathrm{~Gy}$ irradiation, mixed with $0.7 \%$ low melting point agarose, and plated on CometSlide microscope slides, followed by cell lysis. Cells then subjected to electrophoresis under neutral conditions and were stained with SYBR Gold (S11494, Thermo Fisher Scientific). Comets were visualized using a Zeiss fluorescent microscope and were scored (100 cells per treatment) followed by analysis using CASP Comet Assay Software.

2.7. Chromatin immunoprecipitation assay. Cells were cross-linked with $4 \%$ formaldehyde for 10 min followed by cell collection and sonication with a predetermined power to yield genomic DNA fragments 300-1000 bp in length. Lysates were precleared sequentially with normal rabbit IgG (\#sc-2027, Santa Cruz Biotechnology) and Protein A/G. Anti-ARv7 antibody $(2.0 \mu \mathrm{g})$ was added to the cell lysates and incubated at $4^{\circ} \mathrm{C}$ overnight. As a negative control, IgG was used in the reaction. Specific primer sets were designed to amplify a target sequence within the human QKI gene promoter. PCR products were analyzed by agarose gel electrophoresis.

2.8. Luciferase reporter assay. The human promoter region of the QKI 5' promoter was constructed into the pGL3-basic vector (Promega). Site-directed mutagenesis of the ARv7 binding site in the NHS 5' promoter was achieved with the Quick-Change mutagenesis kit. C4-2 -IRR cells were plated in 24-well plates, and the cDNAs were transfected with Lipofectamine 3000 transfection reagent (Invitrogen) according to the manufacturer's instructions. PRL-TK was used as an internal control that served as the baseline control response. Luciferase activity was measured 48 hours after transfection by the DualLuciferase Reporter Assay System (Promega) according to the manufacturer's manual.

2.9. Pull-down assay with biotinylated circNHS probe. In brief, $1 \times 10^{7} \mathrm{C} 4-2-I R R$ cells were harvested, lysed, and sonicated. The cell lysate mixture was rotated overnight at $4^{\circ} \mathrm{C}$ after adding $2 \mu \mathrm{L}$ RNase inhibitor and $500 \mathrm{pmol} / \mathrm{L}$ biotin-labeled antisense oligo against circNHS. The lysate mixture was rotated 
for 2 hours at $4^{\circ} \mathrm{C}$ after adding $10 \mu \mathrm{L}$ streptavidin agarose beads (No. 88817, Pierce Biotechnology). Total RNA was extracted by TRIzol (Invitrogen) according to the manufacturer's protocol, reverse transcribed, and subjected to qPCR analysis to detect the miRNAs.

2.10. RNA in situ hybridization. The biotin-labeled circNHS probe was designed and synthesized by IDT (Integrated DNA Technologies, USA), and the probe sequence is available upon request. The probe signal

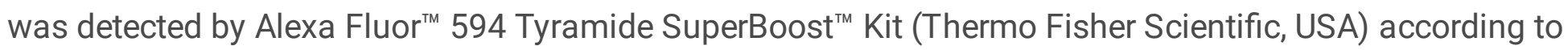
the manufacturer's instructions.

2.11. RNA isolation from nuclear and cytoplasmic fractions. The subcellular localization of circNHS was detected using the PARIS ${ }^{\text {TM }}$ Kit according to the manufacturer's protocol (Thermo Fisher Scientific, USA). 2.12. Actinomycin D assay. C4-2-IRR and 22Rv1 cells were seeded at $1 \times 10^{5}$ cells per well in a 6 -well plate overnight and then exposed to $2 \mathrm{mg} / \mathrm{L}$ actinomycin D (Sigma, USA) for $6,12,18$ and 24 hours. The cells were harvested at the indicated time points, and the stability of circNHS was analyzed using qRTPCR.

2.13. Immunofluorescence. For immunofluorescence, the transfected cells were seeded on glass coverslips for $24 \mathrm{~h}$ and then incubated with primary antibodies against $\mathrm{y}-\mathrm{H} 2 \mathrm{AX}$ at $4^{\circ} \mathrm{C}$ overnight. Cell nuclei were counterstained with $2 \mu \mathrm{g} / \mathrm{ml}$ DAPI for $5 \mathrm{~min}$. Images were captured with a microscope using 20x objectives.

2.14. Xenograft mouse models. To test the effects of the combination of quercetin and IR in tumor growth in vivo, we used the ARv7 positive 22Rv1 cell lines to generate human xenograft tumors in nude mice. 22Rv1 cells $\left(1 \times 10^{6}\right)$ were injected subcutaneously into the left flanks of 6-week-old nude mice (National Cancer Institute). Xenografts reaching approximately $150 \mathrm{~mm}^{3}$, mice were randomized into four groups (four mice/group): (a) vehicle alone (NC group), (b) 2 Gy radiation alone (IR group), (c) $75 \mathrm{mg} / \mathrm{kg}$ quercetin alone (Que group), (d) $75 \mathrm{mg} / \mathrm{kg}$ quercetin combined with irradiation (IR + Que). The groups (b) and (d) animals were given a single dose of quercetin by i.p. injection $6 \mathrm{~h}$ before irradiation. For the irradiation of tumors (group b and d), mice were anesthetized i.p. with Avertin (when irradiated) or isoflurane (when measuring tumor size). Xenografts were locally irradiated with a Model 8114600 Curie Shepherd Cs137 irradiator (URMC, Rochester, NY), while other body parts were protected with lead shielding. Tumor sizes were measured with calipers every 7 days for 28 days, and tumor volumes were estimated using the following formula: volume $=$ (length $\mathrm{x}$ width $\mathrm{x}$ width) $/ 2$. All animal experiments were performed in accordance with the guidelines and with the approval of the University of Rochester Department of Laboratory Animal Medicine.

2.15. Statistical analysis. Unless otherwise stated, all data are shown as the mean \pm standard error of the mean (SEM). Statistical analyses were performed using SPSS 23.0 statistical software. Repeated measures analysis of variance (ANOVA) with Bonferroni correction for comparisons between multiple groups and Student $t$ test for comparisons between two groups were used to determine significance. Significance thresholds are indicated in the Figure legends.

\section{Table 1 Quantitative RT-PCR primers}




\begin{tabular}{|c|c|c|}
\hline ARv7 & Forward & CCATCTTGTCGTCTTCGGAAATGTTA \\
\hline & Reverse & TTTGAATGAGGCAAGTCAGCCTTTCT \\
\hline NHS & Forward & CAGCAGCGCAACATCTTCC \\
\hline & Reverse & CGGTCCTTAAACTCACGCCT \\
\hline GAPDH & Forward & GGAGCGAGATCCCTCCAAAAT \\
\hline & Reverse & GGCTGTTGTCATACTTCTCATGG \\
\hline hsa_circ_0000119 & Forward & TTGGCTGAGAAACTCCTTCC \\
\hline & Reverse & GCTTCTTCCAAGGCCTTCTC \\
\hline hsa_circ_0000518 & Forward & GCCCTAACAGGGCTCTCC \\
\hline & Reverse & AAGGGACATGGGAGTGGAGT \\
\hline hsa_circ_0000554 & Forward & GGCACTAGGGAGGGACTCAT \\
\hline & Reverse & GGGCAGAGACAGAGTGGATG \\
\hline hsa_circ_0001313 & Forward & CGAGACAGACGACGACAAAA \\
\hline & Reverse & TTGACGGTCATCTTCTATTTGC \\
\hline hsa_circ_0002019 & Forward & GGCTTCAGAAAAGGATGCTG \\
\hline & Reverse & TGAAAAGAGGGAGAGCTGGA \\
\hline hsa_circ_0004370 & Forward & CAGGAGACGTGACTGCTGTG \\
\hline & Reverse & TGTCCTATTCCTTCGCTGCT \\
\hline hsa_circ_0004519 & Forward & TGGCATACTGTGATGCTCTG \\
\hline & Reverse & CGGCACTGTGCTGATTCTAA \\
\hline hsa_circ_0007380 & Forward & GTTAGCATGATCCCACCACTG \\
\hline & Reverse & TTTTCCAAGTGTGGCGATTT \\
\hline hsa_circ_0007613 & Forward & GTCCCGTGTGGTCCGAAA \\
\hline & Reverse & CTGCCGTCCTTCACAACTTC \\
\hline hsa_circ_0008301 & Forward & CTTCCAGCTGCCATGGTG \\
\hline & Reverse & CGTGATGCGGAGGAAGTC \\
\hline hsa_circ_0013737 & Forward & GGAGGCCTACTTGCAGCATA \\
\hline & Reverse & ССАTCTTTGCCCATCTAGGA \\
\hline
\end{tabular}




\begin{tabular}{cll} 
hsa_circ_0014879 & Forward & GGCACTGGAACCAGACTCTC \\
\hline & Reverse & GTAGCCAGCCTTTCCTCCTT \\
\hline hsa_circ_0029853 & Forward & GACCTTGCAAAGGAGGTTCA \\
\hline & Reverse & ATCTTGGGGGAATGAAGAGG \\
\hline hsa_circ_0032832 & Forward & TCATCAATGCTGAGGAGCAG \\
\hline hsa_circ_0035381 & Forward & CTGTGCTGTGGACACTGCTT \\
\hline hsa_circ_0058058 & Forward & GCAGAGCTCCGAGAGTAAGG \\
\hline & Reverse & GAAGCGACCAGATTCAAACC \\
\hline hsa_circ_0061137 & Forward & GCCAGAAAAGACTGCTGCTC \\
\hline & Reverse & GTTGTCAGCCACTGTTCACG
\end{tabular}

\section{Results}

\subsection{IR-induced ARv7 expression led to decrease radiosensitivity}

Recent studies indicated that ADT treatment would induce the expression of ARv7 $(23,24)$,the potential impact of IR treatment on ARv7 expression, however, remains unclear. Here we first developed the radioresistant C4-2-IRR (C4-2-lonizing Radiation Resistance) cell line via treating parental C4-2 cells with repeated $2 \mathrm{~Gy}$ radiation. Radioresistance of cells were confirmed in clonogenic assays by showing higher survival of C4-2-IRR cells than respective parental cells at indicated radiation dose (Fig. S1A).

Interestingly, we found that IR could increase significantly the ARv7 expression at the protein and mRNA levels after IR in ARv7-positive 22Rv1 cells and 22Rv1 xenografts (Fig. 1A and Fig. 1B).

To further examine if IR may function via inducing the ARv7 to increase the radioresistance, we first added 5um enzalutamide to inhibit AR activity, then knocked down ARv7 with shARv7 in C4-2-IRR cells or ectopic overexpression of ARv7 in C4-2 cells. The results revealed that suppressed ARv7 in C4-2-IRR cells led to increase the PCa radiosensitivity (Fig. 1C). In contrast, increased ARv7 in C4-2 cells can then increase the radioresistance of PCa cells (Fig. 1D). As 22Rv1 cells have both ARfl and ARv7, we then applied the shAR and/or shARv7 to suppress the expression of ARfl and/or ARv7. The results revealed such suppression to ARfl alone or ARv7 alone could not led to decrease clonogenic survival following IR (Fig. 1E), and only knockdowning of both ARfl and ARv7 led to decrease the clonogenic survival following IR (Fig. 1E). Together, these results suggest that suppression both ARfl and ARv7 could lead to better effect to improve PCa radiosensitivity. 
To further strengthen above conclusion, we added 5um enzalutamide to inhibit AR activity, then knocked down ARv7 with shARv7 in C4-2-IRR cells or ectopic overexpression of ARv7 in C4-2 cells. Next, we examined the ARv7 potential impact on PCa cells DDR by comet assays and the results from these assays also revealed that decreasing ARv7 in C4-2-IRR cells significantly increased DNA fragmentation following IR (Fig. 1F). Similar results from WB (Fig. 1G, left) analysis of the $\mathrm{Y}-\mathrm{H} 2 \mathrm{AX}$ levels showed that decreasing ARv7 in C4-2-IRR cells increased the DNA damage repair time. In contrast, increasing ARv7 resulted in less time to complete DDR in the C4-2 cells (Fig. 1G, right).

Together, results from Fig. 1 suggest that IR-induced ARv7 may decrease the continue RT efficacy.

3.2. Mechanism dissection of how ARv7 can alter the radiosensitivity: via increasing circNHS expression.

To further dissect the mechanism of how ARv7 can alter the radiosensitivity, we focused on circRNAs, as recent studies indicated that the expression of selected circRNAs could be altered after IR exposure (20, 21). From circRNA Array data in radioresistant cancer cells (25), we first chose the 10 most upregulated/downregulated circRNAs and detected their expression in C4-2-IRR cells model. The results revealed that only 7 of those 20 circRNAs had changed significantly compared with C4-2 parental cells (Fig. 2A), and PCR results revealed that decreasing ARv7 led to significantly decreased expression of circNHS (hsa_circ_0089974) in C4-2-IRR cells (Fig. 2B). In contrast, increasing ARv7 led to significantly increased expression of circNHS in C4-2 cells (Fig. 2C). Moreover, we found that IR could increase significantly circNHS levels in 22Rv1 xenografts (Fig. 2D).

Next, results from circBase database analysis indicated that circNHS is generated from back-splicing of two exons (exons 2 and 3) of the NHS gene (chrX:17705861-17710588) (Fig. 2E). To avoid trans-splicing or genomic rearrangements, including head-to-tail splicing, we then applied multiple approaches to rule out such possibilities. We first designed convergent primers to amplify NHS mRNA and divergent primers to amplify circNHS. Using cDNA and genomic DNA from C4-2-IRR and 22Rv1 cell lines as templates, circNHS was amplified from cDNA only by the divergent primers, whereas no amplification product was observed from genomic DNA (Fig. 2F). Furthermore, the results from the RNase R assay revealed that circNHS is the circular form, with better resistance to RNase R digestion than linear NHS (Fig. 2G). Finally, the results from adding actinomycin $D$ to inhibit transcription also indicated that the half-life of circNHS is more stable than NHS mRNA in C4-2-IRR and 22Rv1 cells (Fig. 2H). Finally, the circular RNA microarrays data from Yang et al (26) showed that circNHS were highly expressed in high-grade (Gleason >8) PCa tissues when compared with low-grade (Gleason <6) PCa tissues (Fig. S1B).

Together, the results from Fig. 2 suggest that ARv7 may decrease radiosensitivity by increasing circNHS expression.

\subsection{ARv7 increases circNHS expression via altering the transcriptional regulation}


To further dissect the molecular mechanism of how ARv7 can increase circNHS expression, we examined circNHS transcriptional regulation, and our data indicated that ARv7 could increase NHS expression at both the mRNA and protein levels (Fig. S1C and Fig. S1D). We then searched for potential androgen response elements (AREs) on the NHS 5' promoter region using the JASPAR database. The results revealed that 2 putative AREs were located within $2 \mathrm{~kb}$ of the NHS 5 ' promoter region (Fig. S1E). We then performed chromatin immunoprecipitation (ChIP) in vivo binding assays in 22Rv1 cells, and the results revealed that ARv7 could bind to the first ARE (Fig. 3A), suggesting that ARv7 might be able to increase circNHS expression via direct binding to a ARE to exert its transcriptional regulation.

We then performed the luciferase reporter assay by inserting the $1 \mathrm{~kb} 5$ ' promoter region of NHS containing ARE1 into the pGL3 luciferase backbone and also generated a version with a mutated ARE1 (Fig. S1F). The luciferase assay results revealed that decreasing ARv7 by adding ShARv7 significantly decreased luciferase activity in 22Rv1 transfected with the wild-type NHS promoter construct but not in cells with the mutant NHS promoter construct (Fig. 3B). Importantly, the results from the LinkedOmics database via TCGA data analysis also showed that NHS expression was positively correlated with AR expression in PCa patients (Fig. S1G).

Together, results from Fig. 3A-B suggest that ARv7 increases circNHS expression via altering the transcriptional regulation

Next, to prove that ARv7 may function by altering circNHS expression to decrease radiosensitivity, we then suppressed the circNHS with the shcircNHS, and results revealed that suppressed circNHS (Fig. S1H) led to increase the radiosensitivity in C4-2-IRR cells (Fig. 3C). In contrast, increasing circNHS resulted in decreasing the radiosensitivity in C4-2 cells (Fig. 3D).

The results from the $\mathrm{y}-\mathrm{H} 2 \mathrm{AX}$ assay also revealed that decreasing circNHS levels could increase the DNA damage repair time in C4-2-IRR cells (Fig. 3E, left panel). In contrast, increased circNHS expression resulted in less time to complete DNA damage repair in the C4-2 cell lines (Fig. 3E, right panel). Similarly, the results from the comet assay also revealed that decreasing circNHS in C4-2-IRR cells significantly increased DNA fragmentation following IR (Fig. 3F).

Finally, the results from the silencing experiment revealed that suppressing circNHS led to a partial reversal of ectopic overexpression of ARv7-suppressed radio-sensitivity in the C4-2-IRR cells (Fig. 3G) and increased circNHS led to a partial reversal of the shARv7-increased radio-sensitivity in the C4-2 cells (Fig. 3H).

Together, results from Fig. 3 suggest that ARv7 can function via increasing the circNHS to alter the radiosensitivity.

\subsection{Mechanistic dissection of how the circNHS could decrease radiosensitivity: by competing with miR-512- $5 \mathrm{p} / \mathrm{XRCC} 5$ axis}


Next, to determine how circNHS could decrease radiosensitivity, we first determined the subcellular localization of circNHS in PCa cell lines using a nuclear mass separation assay (Fig. 4A) and FISH analysis (Fig. 4B). The results revealed that circNHS was expressed mainly in the cytoplasm of PCa cells. As early studies indicated that cytoplasmic circRNAs might compete with miRNAs to exert their function (27), we then performed an RNA immunoprecipitation (RIP) assay with an antibody against argonaute 2 (AGO2) in C4-2-IRR and 22Rv1 cells, and the results revealed that circNHS was significantly enriched by the AGO2 antibody (Fig. 4C), suggesting that circNHS might act as a binding platform for AGO2 and miRNAs. Based on these findings, we predicted that circNHS might serve as a binding platform for miRNAs. We then surveyed 3 databases $(28,29)$ and found that 16 potential miRNAs could bind to circNHS (Fig. 4D). Using probes specifically against circNHS to analyze these 16 candidate miRNAs in the complex, we found only a specific enrichment of miR-512-5p and none of the rest 15 miRNAs, suggesting that miR-512-5p is one of the circNHS-associated miRNAs in PCa cells.

To further prove that the circNHS can alter radiosensitivity via binding to the miR-512-5p, we then constructed a mutated binding site of miR-512-5p (circNHS-Mut, Fig. S1I). The results from colony formation assays revealed that mutation of the miR-512-5p binding site could completely abolish circNHS-induced radiosensitivity in C4-2 cells (Fig. 4E).

Recent studies identified 542 ARv7-regulated genes via RNA-seq and CHIP-seq analyses (30). Among these genes, we found 9 DDR genes that may involve in DNA damage repair, and results from targetscan database predicted that XRCC5 and SPRTN can be the potential target gene of miR-512-5p (Fig. 4F). Results from western blot analysis demonstrated that circNHS could regulate the expression of XRCC5 protein, and not SPRTN protein levels, suggesting circNHS may function via sponging miR-512-5p to increase the protein expression of XRCC5 (Fig. 4G). As expectedly, adding miR-512-5p inhibitor led to increase XRCC5 protein expression and could partly reverse the shcircNHS-decreased XRCC5 expression in C4-2 cells (Fig. 4H, left) while adding miR-141-3p mimics led to decreased XRCC5 protein expression and could effectively reverse the oe-circNHS increased XRCC5 expression in C4-2-IRR cells (Fig. 4H, right).

Results from interruption approaches using XRCC5-shRNA further revealed that suppressing XRCC5 led to partially reverse the ARv7-increased radioresistance (Fig. 4I). Similarly, adding XRCC5-cDNA led to partially reverse the shARv7-decreased the radioresistance in C4-2-IRR cells (Fig. 4J).

Finally, the results from the LinkedOmics database via TCGA data analysis also showed that XRCC5 expression was positively correlated with AR expression in PCa patients (Fig. S1J).

Together, the results from Fig. 4 suggest that circNHS might function by sponging miR-512-5p to alter XRCC5 protein expression and such regulation may then lead to alter the DDR pathway.

\subsection{Preclinical studies in in vitro cell lines and in in vivo mouse model to target ARv7-mediated circNHS/miR-512-5p/XRCC5 signaling with small molecule of Que to increase radiosensitivity}


All above results suggest that IR may have unwanted side effects of inducing the ARv7 expression to increase the radioresistance. We are interested to see if adding small molecules can have similar effect to suppress the ARv7 expression. We are interested to test the Que, as our previous studies indicated that Que could suppress AR mRNA and protein expression (31). Here we found that adding Que could also significantly decrease the ARv7 expression at both mRNA and protein levels in 22Rv1 and VCaP cells (Fig. 5A-C).

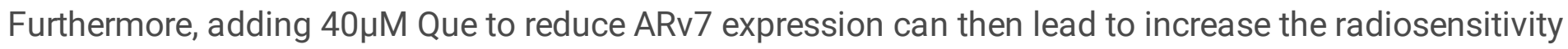
to better suppress the C4-2-IRR and 22Rv1 cell growth using clonogenic assay (Fig. 5D-E). Mechanism dissection revealed that adding Que, and not Enz, could decrease DNA damage repair via detecting $Y^{-}$ H2AX levels (Fig. 5F).

To further validate these in vitro findings in the in vivo mouse model, we established subcutaneous 22Rv1 xenografts in nude mice. Results showed that combined 2Gy IR treatment with Que treatment $(75 \mathrm{mg} / \mathrm{kg})$ led to suppress the tumor growth approximately $70-75 \%$ on day 28 , as compared to 2 Gy IR alone or Que alone (Fig. 5G).

Together, results from Fig. 5 suggest that small molecule of Que can increase radiosensitivity to better suppress the PCa cells growth via targeting both ARfl and ARv7 levels.

\section{Discussion}

Studies reported that AR-FL mRNA is coded from eight exons and the protein contains four functional domains: an N-terminal domain (NTD), a central DNA-binding domain (DBD), a short hinge region and a C-terminal LBD (32). Constitutively active ARVs have now been discovered (33), most of which contain the NTD and the DBD, but lack the LBD owing to a truncation by a cryptic exon. As conventional ADT inhibits androgen-dependent activation of $A R$, the presence of C-terminal truncated ARVs provides a compelling mechanism for CRPC cells to circumvent ADT (34). ARVs are nuclear, constitutively active, bind to similar ARE, as that engaged by full-length AR (34). Among $>20$ different ARVs that have been identified in clinical PC and PC cell lines and among these variants, AR-V7, originates from contiguous splicing of AR exons 1, 2, and 3 with the cryptic exon 3 (CE3) present within the canonical intron 3 of the AR gene, is one of the most well characterized $A R-V s$ and can be reliably measured in tissue and liquid biopsy specimens, and blood-based detection (35-38). Owing to lack of LBD, AR-V7 can circumvent the pharmacological effects of second-generation antiandrogens such as enzalutamide, which targets LBD of AR directly. Besides,ARv7 could be induced by ADT treatment and also serves as a predictive biomarker for response to enzalutamide or abiraterone acetate $(7,9,23)$.

We and some groups have demonstrated that AR expression and activity are durably upregulated following IR in PCa models and AR can activate DDR pathways $(4,5,10,39,40)$ in PCa, providing rationale for concurrent ADT + RT therapy $(6,41)$ is better than RT therapy alone. These preclinical studies also have led to testing combinations of RT with second-generation antiandrogens such as abiraterone, enzalutamide, apalutamide and AR degradation enhancer, ASC-J9 (40, 42-45). However, despite use of 
combined ADT and RT, the recurrence rate is up to $50 \%$ of high-risk PCa patients (6). To dissect this mechanism, we focus on the role of ARv7 expression and activity in PCa after RT and radioresistant PCa cells. Our in vitro and in vivo experiments results suggest that ARv7 is highly expressed in radioresistant PCa cells and IR could induce ARv7 expression in PCa which increase the clonogenic survival of PCa cells after irradiation. Moreover, these IR induced ARv7 could mediate PCa DDR and is not affected by enzalutamide treatment. Thus, our study may explain the clinical phenomenon that many patients first respond to RT + ADT then eventually became biochemical disease relapse and that patients with ARv7 expression might not be eligible for such a combination treatment. Similarly, Yin et al. also found that ARVs alone can regulate DDR following PCa RT. To clarify these issues, further investigation on PCa RT clinical samples are necessary.

To further dissect the mechanism how ARv7 may alter the IR-sensitivity, recently studies found that circRNAs may play important roles in radiosensitivity. Several studies have revealed that circRNA levels might be substantially altered after IR, including esophageal cancer (3), cervical cancer (46), glioma (47), nasopharyngeal carcinoma (48), oral squamous cell carcinoma (49), colorectal cancer (50) and hepatocellular carcinoma (51). Previously, we found that circZEB1 was upregulated after IR in PCa cells and might function as a sponge for miR-141-3p (22). In this study, we analyzed our radioresistant C4-2 cell models and analyzed the twenty most altered circRNAs from circRNA-Array data (25). Studies showed that AR-V7 is constitutively active and capable of activating genes even in the absence of androgen (52). Our results showed that only circNHS could be regulated by ARv7 among these twenty circRNAs. By gain/loss of function assays of circNHS, we found that it could partially reverse the effects of ARv7 on PCa radiosensitivity. Moreover, there are currently no other published reports about circNHS functions now. To our knowledge, this is the first study about circRNA function in radioresistant PCa cells to date, which might contribute to a better understanding of ARv7 function in RT.

Taken together, a requirement for inhibiting both full-length AR and ARv7 transcriptional activities is necessary to effectively improve radiosensitivity. To target the IR-induced both AR and ARv7 with small molecules, we screened and found that a small molecule Quercetin which is a flavonoid widely found in vegetables and fruits particularly in onions, apples, and red wine (12). Moveover, quercetin is of very low toxicity and rarely produce any side effects even at high dose of $200 \mathrm{mg} / \mathrm{kg}$ given to rats and mice (53, 54 ), and clinical trial showed that total consumption of $1000 \mathrm{mg}$ quercetin per day could be well tolerated in human not associated with any side effects $(55,56)$. Recent studies have found its antitumor role in many cancers and can function as radiosensitizer in many cancers (57-59). Our previous study also revealed that quercetin inhibited $\mathrm{LNCaP}$ cells growth through inhibiting androgen receptor expression and its inducible genes (31). In this study, we found that quercetin could inhibit the transcriptional activities of both full-length AR and ARv7 and enhance RT-mediated cell killing compared with second-generation antiandrogens such as enzalutamide. Thus, we provide proof-of-principle pre-clinical in vitro and in vivo evidence to rationalize the clinical use of quercetin to enhance the effect of IR as a potential strategy to improve the outcomes of PCa patients who undergo RT. Indeed, further randomized clinical trials are required to assess the effect of combining Que and RT for PCa patients. 


\section{Conclusions}

In summary, our research here demonstrated that IR could induced ARv7 in PCa cells and IR-induced ARv7 could decrease the radiosensitivity through mediating circNHS/miR-512-5p/XRCC5 pathway. Targeting the IR-induced ARv7 and AR with small molecule of quercetin could increase radiosensitivity to better suppress the PCa cells. These findings can provide a solid foundation for the clinical use of quercetin in RT and raise the possibility of applying combined use of RT and quercetin to gain better clinical results than $\mathrm{RT}+\mathrm{ADT}$ treatment.

\section{Abbreviations}

ceRNA

Competing endogenous RNA; CircRNA:Circular RNA; PCa:prostate cancer; qRT-PCR:Real-time quantitative polymerase chain reaction; miRNA:MicroRNA; RIP:RNA immunoprecipitation; RNA-seq:RNA sequencing; TCGA:The Cancer Genome Atlas; Que:Quercetin; ARv7:androgen receptor variants 7.

\section{Declarations}

\section{Acknowledgment:}

We thank Ms. Karen Wolf for helping with the preparation of the manuscript.

\section{Authors' contributions}

Conception and design: CSC, NZX; Development of methodology: DC, YCC, YJN, SYY; Acquisition of data: DC, FJC, YCC, YQW, CPH, TH; Analysis and interpretation of data: DC, FJC, YCC; Writing, review, and revision of the manuscript: DC, CSC, NZX; Administrative, technical, or material support: SYY, CSC, NZX; Study supervision: CSC, NZX

\section{Funding}

This work was supported by the George Whipple Professorship, the National Natural Science Foundation of China (Project number: 81772700 and 81972400), the Beijing Capital Science and Technology Leading Talent Project (Project number: Z181100006318007) and CAMS Innovation Found for Medical Sciences (Project number: 2019-I2M-1-003).

\section{Availability of data and materials}

The datasets used and/or analyzed during this study are available from the corresponding author on reasonable request.

\section{Ethics approval and consent to participate}


The ethics approval statements for animal work were provided by University of Rochester Department of Laboratory Animal Medicine.

\section{Consent for publication}

All authors give consent for the publication of the manuscript in Journal of Experimental \& Clinical Cancer Research.

\section{Competing interests}

The authors declare that they have no competing interests.

\section{References}

1. Hayden AJ, Catton C, Pickles T. Radiation therapy in prostate cancer: a risk-adapted strategy. Current oncology. 2010;17(Suppl 2):18-24.

2. Zhu Y, Wang HK, Qu YY, Ye DW. Prostate cancer in East Asia: evolving trend over the last decade. Asian journal of andrology. 2015;17(1):48-57.

3. Sanda MG, Cadeddu JA, Kirkby E, Chen RC, Crispino T, Fontanarosa J, et al. Clinically Localized Prostate Cancer: AUA/ASTRO/SUO Guideline. Part II: Recommended Approaches and Details of Specific Care Options. The Journal of urology. 2018;199(4):990-7.

4. Goodwin JF, Schiewer MJ, Dean JL, Schrecengost RS, de Leeuw R, Han S, et al. A hormone-DNA repair circuit governs the response to genotoxic insult. Cancer discovery. 2013;3(11):1254-71.

5. Polkinghorn WR, Parker JS, Lee MX, Kass EM, Spratt DE, laquinta PJ, et al. Androgen receptor signaling regulates DNA repair in prostate cancers. Cancer discovery. 2013;3(11):1245-53.

6. Bolla M, Van Tienhoven G, Warde P, Dubois JB, Mirimanoff RO, Storme G, et al. External irradiation with or without long-term androgen suppression for prostate cancer with high metastatic risk: 10year results of an EORTC randomised study. The Lancet Oncology. 2010;11(11):1066-73.

7. Yu Z, Chen S, Sowalsky AG, Voznesensky OS, Mostaghel EA, Nelson PS, et al. Rapid induction of androgen receptor splice variants by androgen deprivation in prostate cancer. Clinical cancer research: an official journal of the American Association for Cancer Research. 2014;20(6):1590-600.

8. Sun S, Sprenger CC, Vessella RL, Haugk K, Soriano K, Mostaghel EA, et al. Castration resistance in human prostate cancer is conferred by a frequently occurring androgen receptor splice variant. The Journal of clinical investigation. 2010;120(8):2715-30.

9. Beltran H, Wyatt AW, Chedgy EC, Donoghue A, Annala M, Warner EW, et al. Impact of Therapy on Genomics and Transcriptomics in High-Risk Prostate Cancer Treated with Neoadjuvant Docetaxel and Androgen Deprivation Therapy. Clinical cancer research: an official journal of the American Association for Cancer Research. 2017;23(22):6802-11.

10. Yin Y, Li R, Xu K, Ding S, Li J, Baek G, et al. Androgen Receptor Variants Mediate DNA Repair after Prostate Cancer Irradiation. Cancer research. 2017;77(18):4745-54. 
11. Massi A, Bortolini O, Ragno D, Bernardi T, Sacchetti G, Tacchini M, et al. Research Progress in the Modification of Quercetin Leading to Anticancer Agents. Molecules. 2017;22:8.

12. Bischoff SC. Quercetin: potentials in the prevention and therapy of disease. Current opinion in clinical nutrition metabolic care. 2008;11(6):733-40.

13. Kim GT, Lee SH, Kim JI, Kim YM. Quercetin regulates the sestrin 2-AMPK-p38 MAPK signaling pathway and induces apoptosis by increasing the generation of intracellular ROS in a p53independent manner. International journal of molecular medicine. 2014;33(4):863-9.

14. Chan ST, Yang NC, Huang CS, Liao JW, Yeh SL. Quercetin enhances the antitumor activity of trichostatin A through upregulation of p53 protein expression in vitro and in vivo. PloS one. 2013;8(1):e54255.

15. Piao S, Kang M, Lee YJ, Choi WS, Chun YS, Kwak C, et al. Cytotoxic effects of escin on human castration-resistant prostate cancer cells through the induction of apoptosis and G2/M cell cycle arrest. Urology. 2014;84(4):982 e1-7.

16. Li X, Yang L, Chen LL. The Biogenesis, Functions, and Challenges of Circular RNAs. Molecular cell. 2018;71(3):428-42.

17. Zhang Z, Yang T, Xiao J. Circular RNAs: Promising Biomarkers for Human Diseases. EBioMedicine. 2018;34:267-74.

18. Han B, Chao J, Yao H. Circular RNA and its mechanisms in disease: From the bench to the clinic. Pharmacology therapeutics. 2018;187:31-44.

19. Qian L, Yu S, Chen Z, Meng Z, Huang S, Wang P. The emerging role of circRNAs and their clinical significance in human cancers. Biochimica et biophysica acta Reviews on cancer. 2018.

20. Cui C, Yang J, Li X, Liu D, Fu L, Wang X. Functions and mechanisms of circular RNAs in cancer radiotherapy and chemotherapy resistance. Mol Cancer. 2020;19(1):58.

21. Podralska M, Ciesielska S, Kluiver J, van den Berg A, Dzikiewicz-Krawczyk A, Slezak-Prochazka I. Non-Coding RNAs in Cancer Radiosensitivity: MicroRNAs and IncRNAs as Regulators of RadiationInduced Signaling Pathways. Cancers (Basel). 2020;12(6).

22. Chen $D$, Chou FJ, Chen $Y$, Tian $H$, Wang $Y$, You B, et al. Targeting the radiation-induced TR4 nuclear receptor-mediated QKI/circZEB1/miR-141-3p/ZEB1 signaling increases prostate cancer radiosensitivity. Cancer Lett. 2020;495:100-11.

23. Antonarakis ES, Lu C, Wang H, Luber B, Nakazawa M, Roeser JC, et al. AR-V7 and resistance to enzalutamide and abiraterone in prostate cancer. The New England journal of medicine. 2014;371(11):1028-38.

24. Wang R, Sun Y, Li L, Niu Y, Lin W, Lin C, et al. Preclinical Study using Malat1 Small Interfering RNA or Androgen Receptor Splicing Variant 7 Degradation Enhancer ASC-J9((R)) to Suppress Enzalutamideresistant Prostate Cancer Progression. European urology. 2017;72(5):835-44.

25. Su H, Lin F, Deng X, Shen L, Fang Y, Fei Z, et al. Profiling and bioinformatics analyses reveal differential circular RNA expression in radioresistant esophageal cancer cells. Journal of translational medicine. 2016;14(1):225. 
26. Yang Z, Qu CB, Zhang Y, Zhang WF, Wang DD, Gao CC, et al. Dysregulation of p53-RBM25-mediated circAMOTL1L biogenesis contributes to prostate cancer progression through the circAMOTL1L-miR193a-5p-Pcdha pathway. Oncogene. 2019;38(14):2516-32.

27. Bach DH, Lee SK, Sood AK. Circular RNAs in Cancer. Molecular therapy Nucleic acids. 2019;16:11829.

28. Kruger J, RNAhybrid RM. microRNA target prediction easy, fast and flexible. Nucleic acids research. 2006;34(Web Server issue):W451-4.

29. Huang $\mathrm{HY}$, Chien $\mathrm{CH}$, Jen $\mathrm{KH}$, Huang HD. RegRNA: an integrated web server for identifying regulatory RNA motifs and elements. Nucleic acids research. 2006;34(Web Server issue):W429-34.

30. He Y, Lu J, Ye Z, Hao S, Wang L, Kohli M, et al. Androgen receptor splice variants bind to constitutively open chromatin and promote abiraterone-resistant growth of prostate cancer. Nucleic acids research. 2018;46(4):1895-911.

31. Xing N, Chen Y, Mitchell SH, Young CY. Quercetin inhibits the expression and function of the androgen receptor in LNCaP prostate cancer cells. Carcinogenesis. 2001;22(3):409-14.

32. Bain DL, Heneghan AF, Connaghan-Jones KD, Miura MT. Nuclear receptor structure: implications for function. Annual review of physiology. 2007;69:201-20.

33. Dehm SM, Tindall DJ. Alternatively spliced androgen receptor variants. Endocrine-related cancer. 2011;18(5):R183-96.

34. Wadosky KM, Koochekpour S. Androgen receptor splice variants and prostate cancer: From bench to bedside. Oncotarget. 2017;8(11):18550-76.

35. Lu C, Brown LC, Antonarakis ES, Armstrong AJ, Luo J. Androgen receptor variant-driven prostate cancer II: advances in laboratory investigations. Prostate Cancer Prostatic Dis. 2020;23(3):381-97.

36. Shao C, Yu B, Liu Y. Androgen receptor splicing variant 7: Beyond being a constitutively active variant. Life Sci. 2019;234:116768.

37. Brown LC, Lu C, Antonarakis ES, Luo J, Armstrong AJ. Androgen receptor variant-driven prostate cancer II: advances in clinical investigation. Prostate Cancer Prostatic Dis. 2020;23(3):367-80.

38. Luo J, Attard G, Balk SP, Bevan C, Burnstein K, Cato L, et al. Role of Androgen Receptor Variants in Prostate Cancer: Report from the 2017 Mission Androgen Receptor Variants Meeting. European urology. 2018;73(5):715-23.

39. Bartek J, Mistrik M, Bartkova J. Androgen receptor signaling fuels DNA repair and radioresistance in prostate cancer. Cancer discovery. 2013;3(11):1222-4.

40. Chou FJ, Chen Y, Chen D, Niu Y, Li G, Keng P, et al. Preclinical study using androgen receptor (AR) degradation enhancer to increase radiotherapy efficacy via targeting radiation-increased AR to better suppress prostate cancer progression. EBioMedicine. 2019;40:504-16.

41. Jones JS. Radiorecurrent prostate cancer: an emerging and largely mismanaged epidemic. European urology. 2011;60(3):411-2. 
42. Zhang W, Liao CY, Chtatou H, Incrocci L, van Gent DC, van Weerden WM, et al. Apalutamide Sensitizes Prostate Cancer to Ionizing Radiation via Inhibition of Non-Homologous End-Joining DNA Repair. Cancers (Basel). 2019;11(10).

43. Ghashghaei M, Niazi TM, Heravi M, Bekerat $H$, Trifiro M, Paliouras $M$, et al. Enhanced radiosensitization of enzalutamide via schedule dependent administration to androgen-sensitive prostate cancer cells. Prostate. 2018;78(1):64-75.

44. Elsesy ME, Oh-Hohenhorst SJ, Loser A, Oing C, Mutiara S, Kocher S, et al. Second-Generation Antiandrogen Therapy Radiosensitizes Prostate Cancer Regardless of Castration State through Inhibition of DNA Double Strand Break Repair. Cancers (Basel). 2020;12(9).

45. Ghashghaei M, Kucharczyk M, Elakshar S, Muanza T, Niazi T. Combining prostate cancer radiotherapy with therapies targeting the androgen receptor axis. Current oncology. 2019;26(5):e640e50.

46. Yu D, Li Y, Ming Z, Wang H, Dong Z, Qiu L, et al. Comprehensive circular RNA expression profile in radiation-treated HeLa cells and analysis of radioresistance-related circRNAs. PeerJ. 2018;6:e5011.

47. Zhao M, Xu J, Zhong S, Liu Y, Xiao H, Geng L, et al. Expression profiles and potential functions of circular RNAs in extracellular vesicles isolated from radioresistant glioma cells. Oncology reports. 2019;41(3):1893-900.

48. Yang J, Zhu D, Liu S, Shao M, Liu Y, Li A, et al. Curcumin enhances radiosensitization of nasopharyngeal carcinoma by regulating circRNA network. Molecular carcinogenesis. 2020;59(2):202-14.

49. Chen G, Li Y, He Y, Zeng B, Yi C, Wang C, et al. Upregulation of Circular RNA circATRNL1 to Sensitize Oral Squamous Cell Carcinoma to Irradiation. Molecular therapy Nucleic acids. 2020;19:961-73.

50. Wang L, Peng X, Lu X, Wei Q, Chen M, Liu L. Inhibition of hsa_circ_0001313 (circCCDC66) induction enhances the radio-sensitivity of colon cancer cells via tumor suppressor miR-338-3p: Effects of cicr_0001313 on colon cancer radio-sensitivity. Pathology research practice. 2019;215(4):689-96.

51. Yang W, Liu Y, Gao R, Xiu Z, Sun T. Knockdown of cZNF292 suppressed hypoxic human hepatoma SMMC7721 cell proliferation, vasculogenic mimicry, and radioresistance. Cellular signalling. 2019;60:122-35.

52. Hu R, Dunn TA, Wei S, Isharwal S, Veltri RW, Humphreys E, et al. Ligand-independent androgen receptor variants derived from splicing of cryptic exons signify hormone-refractory prostate cancer. Cancer research. 2009;69(1):16-22.

53. Sharmila G, Bhat FA, Arunkumar R, Elumalai P, Raja Singh P, Senthilkumar K, et al. Chemopreventive effect of quercetin, a natural dietary flavonoid on prostate cancer in in vivo model. Clinical nutrition. 2014;33(4):718-26.

54. Ma ZS, Huynh TH, Ng CP, Do PT, Nguyen TH, Huynh H. Reduction of CWR22 prostate tumor xenograft growth by combined tamoxifen-quercetin treatment is associated with inhibition of angiogenesis and cellular proliferation. International journal of oncology. 2004;24(5):1297-304. 
55. Cialdella-Kam L, Nieman DC, Sha W, Meaney MP, Knab AM, Shanely RA. Dose-response to 3 months of quercetin-containing supplements on metabolite and quercetin conjugate profile in adults. The British journal of nutrition. 2013;109(11):1923-33.

56. Shoskes DA, Zeitlin SI, Shahed A, Rajfer J. Quercetin in men with category III chronic prostatitis: a preliminary prospective, double-blind, placebo-controlled trial. Urology. 1999;54(6):960-3.

57. van Rijn J, van den Berg J. Flavonoids as enhancers of x-ray-induced cell damage in hepatoma cells. Clinical cancer research: an official journal of the American Association for Cancer Research. 1997;3(10):1775-9.

58. Luttjeboer M, Lafleur MV, Kwidama ZJ, Van Rijn J, Van Den Berg J, Slotman BJ, et al. Strategies for the analysis of in vitro radiation sensitivity and prediction of interaction with potential radiation modifying agents. International journal of radiation biology. 2010;86(6):458-66.

59. Lagerweij T, Hiddingh L, Biesmans D, Crommentuijn MH, Cloos J, Li XN, et al. A chemical screen for medulloblastoma identifies quercetin as a putative radiosensitizer. Oncotarget. 2016;7(24):3577688.

\section{Figures}

Figure 1

A

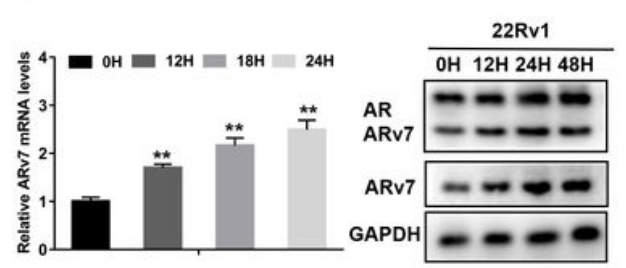

C

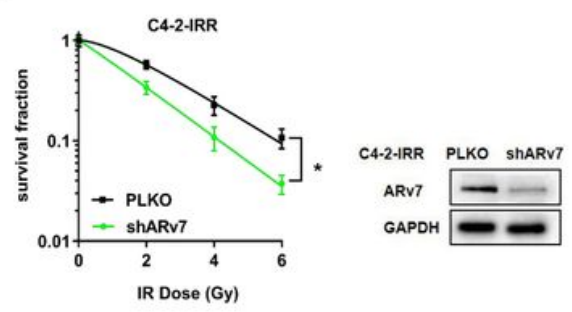

$\mathbf{F}$

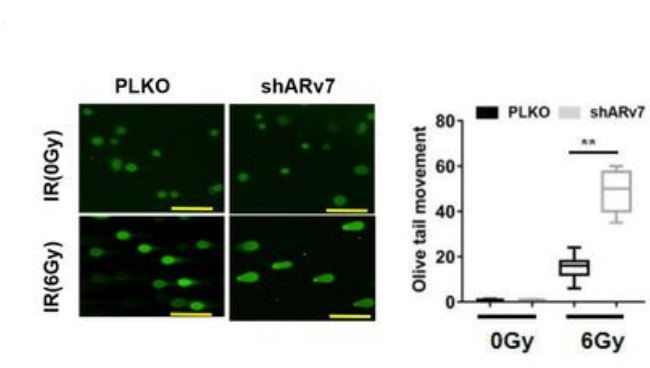

B

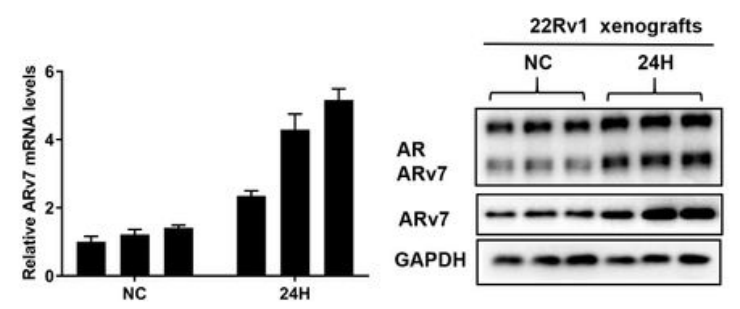

E

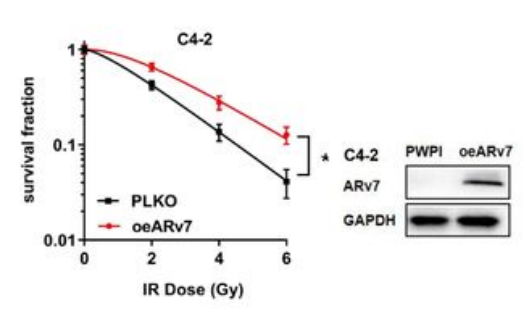

G

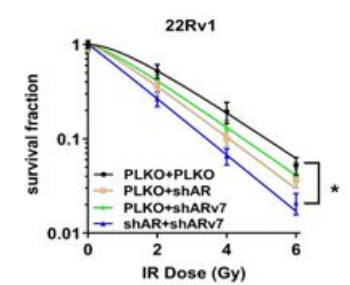

IR Dose (Gy)

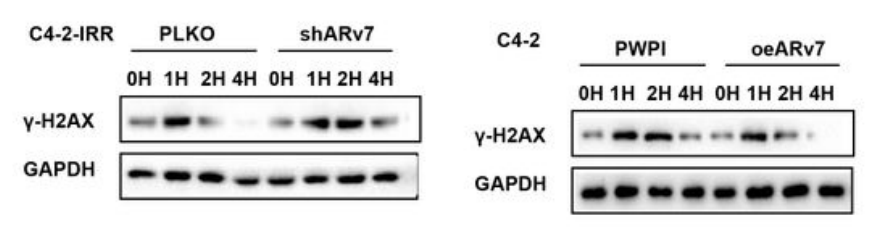

Figure 1

(A) ARv7 mRNA and protein levels in 22Rv1 cells after 4Gy IR treatment. (B) ARv7 mRNA and protein levels in 22Rv1 xenografts after 4Gy IR treatment. (C) C4-2-IRR parental cells and C4-2-IRR-shARv7 cells 
were cultured with $5 \mu \mathrm{M}$ Enz for 24 hours, then treated with escalating doses of IR. (D)The oeARv7 effects on C4-2 cells survival after IR using clonogenic assay. (E) shARv7 and/or shAR effects on 22Rv1 cells survival after IR using clonogenic assay. (F) shARv7 effects on DNA damage in C4-2-IRR cells after IR using comet assay. Scale bar $=10 \mu \mathrm{m}$. $(\mathrm{G})$ Western Blot analysis of $\mathrm{y}-\mathrm{H} 2 \mathrm{AX}$ in different groups. The oeARv7 can decrease DNA damage repair time in C4-2 cells and shARv7 can increase DNA damage repair time in C4-2-IRR cells. Data are presented as mean \pm SEM. ${ }^{*} P<0.05,{ }^{*} \mathrm{P}<0.01$, compared with the controls.

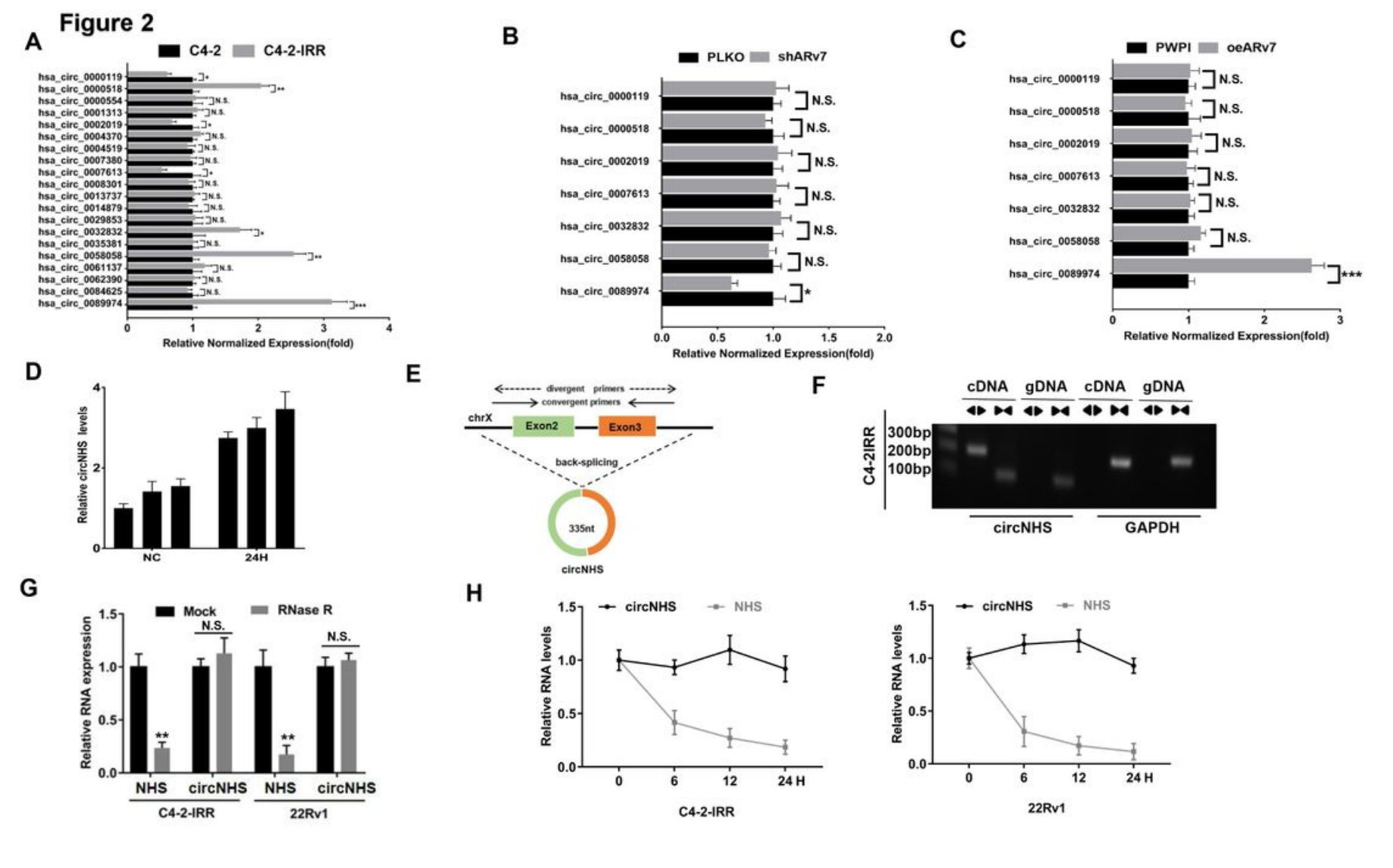

Figure 2

(A) PCR analysis of 20 circRNAs levels in C4-2 parental cells and C4-2-IRR cells. (B) shARv7 in C4-2-IRR cells and PCR analysis of 7 circRNAs levels. (C) oeARv7 in C4-2 parental cells and PCR analysis of 7 circRNAs levels. (D) circNHS levels in 22Rv1 xenografts after 4Gy IR treatment. (E) Schematic diagram of the genomic location and splicing pattern of circNHS. (F) The existence of circNHS was validated in C4-2 parental cells and 22Rv1 cells by PCR. Divergent primers amplified circNHS from cDNA, but not from genomic DNA (gDNA). GAPDH was used as a negative control.Quantitative immunofluorescence of $\gamma^{-}$ H2AX. (G) PCR analysis of RNase R treatment assay to confirm the circNHS formation is a circRNA sequence. $(\mathrm{H})$ The relative RNA levels of circNHS and NHS were analyzed by RT-qPCR after treatment with Actinomycin $D$ at the indicated time points. Data are presented as mean $\pm S E M$. ${ }^{*} P<0.05,{ }^{\star \star} P<0.01$, $\star \star \star P<0.001$ compared with the controls. N.S., not significant compared with the controls. 
A

Figure 3

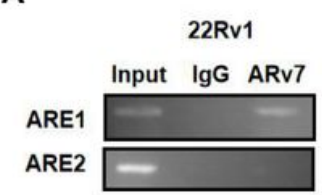

B

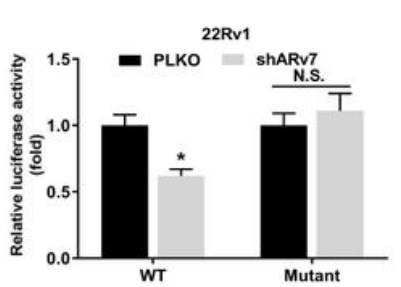

C

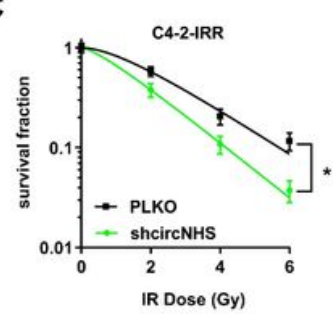

D

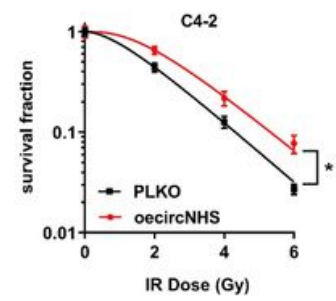

E
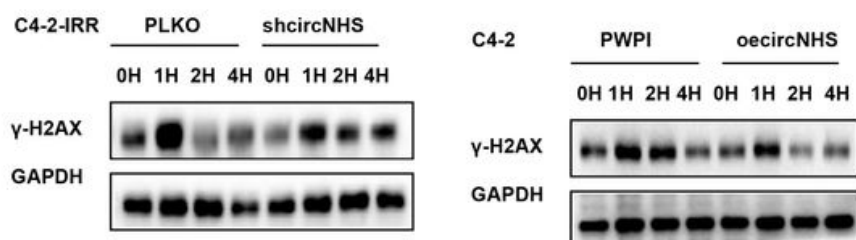

Y-H2AX

GAPDH

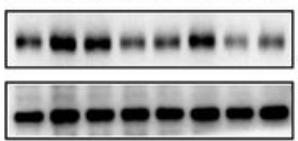

F
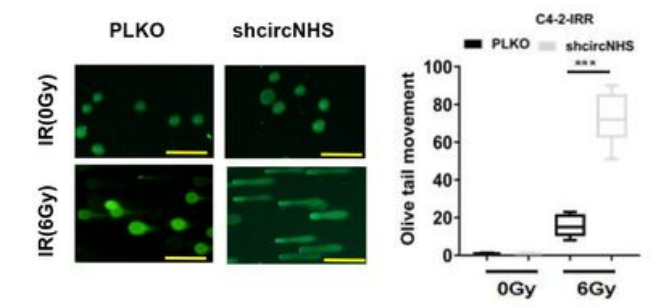

G

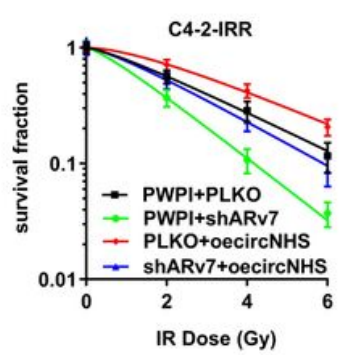

H

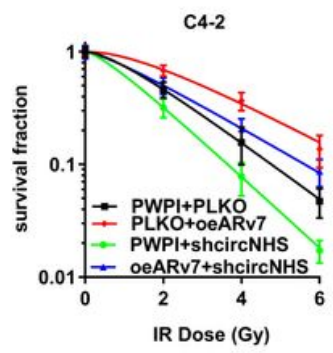

\section{Figure 3}

(A) Chip assay on of the 2 potential ARE binding sites on NHS promoter. (B) Co-transfection of wild type ARE or mutant NHS promoter pGL3-Luciferase constructs into 22Rv1 cells with/without shARv7. The luciferase assay was applied to detect the promoter activity. (C) shcircNHS effects on C4-2-IRR cells survival after IR using clonogenic assay. (D) The oecircNHS effects on C4-2 cells survival after IR using clonogenic assay. (E) Western Blot analysis of $\mathrm{Y}-\mathrm{H} 2 \mathrm{AX}$ in different groups. The oecircNHS can decrease DNA damage repair time in C4-2 cells and shcircNHS can increase DNA damage repair time in C4-2-IRR cells. (F) shcircNHS effects on DNA damage in C4-2-IRR cells after IR using comet assay. Scale bar = $10 \mu \mathrm{m}$. (G) shARv7 and/or oecircNHS effects on C4-2-IRR cells survival after IR using clonogenic assay.

(H) The oeARv7 and/or shcircNHS effects on C4-2 cell survival after IR using clonogenic assay. Data are presented as mean \pm SEM. ${ }^{\star} P<0.05$ and ${ }^{*} * \mathrm{P}<0.01$ compared with the controls. N.S., not significant compared with the controls. 
Figure 4

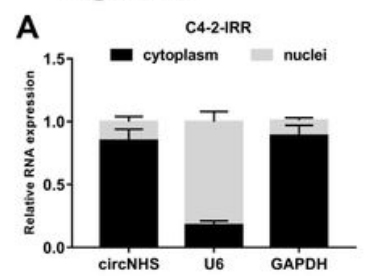

D

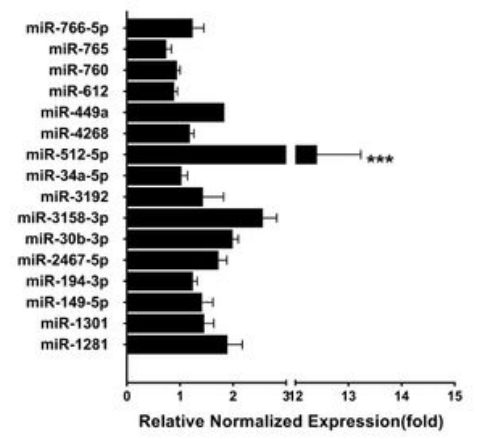

G

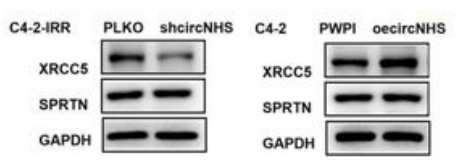

B

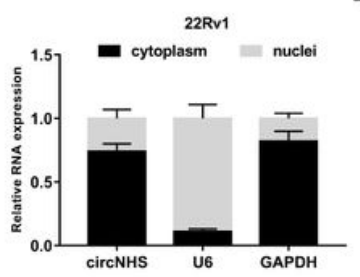

C

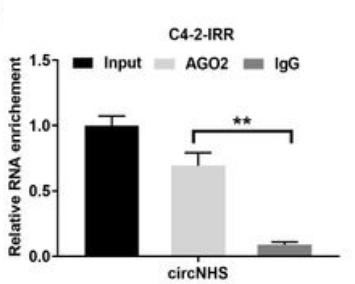

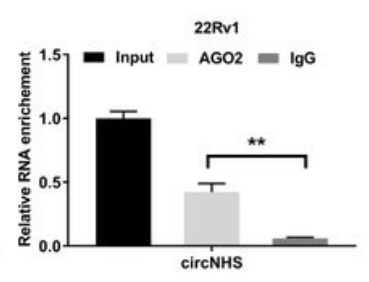

F

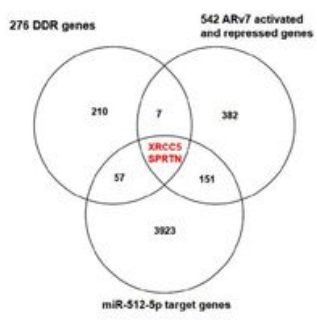

E

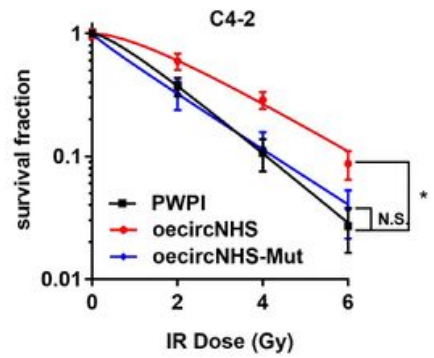

H
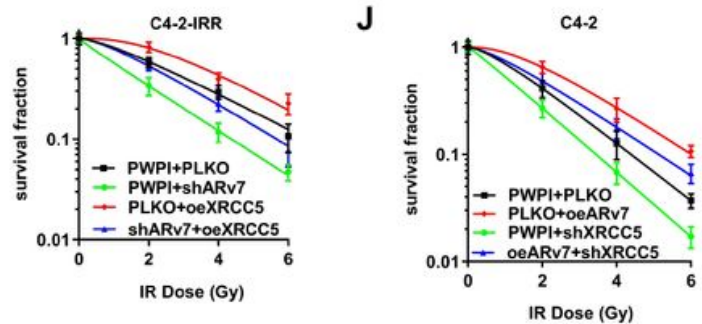

Figure 4

(A) Relative circNHS expression levels in nuclear and cytosolic fractions of C4-2-IRR cells and 22Rv1 cells are shown. U6 was used as the nuclear control. $\beta$-actin was used as the cytosolic control. (B) RNA-FISH indicated the location of circNHS in C4-2-IRR cells and 22Rv1 cells. (C) RIP experiments were performed using an antibody against AGO2 on extracts from C4-2-IRR cells. (D) The circRIP was performed in C4-2IRR cells using a circNHS-specific probe and control probe. The enrichment of miRNAs was detected by RT-qPCR and normalized to the control probe. (E) Mutation of miR-512-5p binding sites abolishes the effects of circNHS on IR sensitivity, as revealed using colony formation assay in C4-2 cells. (F) overlapping of the target genes of circNHS predicted by three datas. (G) Western Blot analysis of XRCC5 and SPRTN protein levels after shcircNHS in C4-2-IRR cells and oecircNHS in C4-2 cells. (H) WB assays: effects of shcircNHS and/or miR-512-5p inhibitor on XRCC5 protein levels in C4-2-IRR cells (left) and effects of oecircNHS and/or miR-512-5p mimics on XRCC5 protein levels in C4-2 cells (right). (I) shARv7 and/or oeXRCC5 effects on C4-2-IRR cells survival after IR using clonogenic assay. (J) The oeARv7 and/or shXRCC5 effects on C4-2 cell survival after IR using clonogenic assay. ${ }^{*} \mathrm{P}<0.01$ compared with the controls. N.S., not significant compared with the controls. Data are presented as mean \pm SEM. 
A

Figure 5

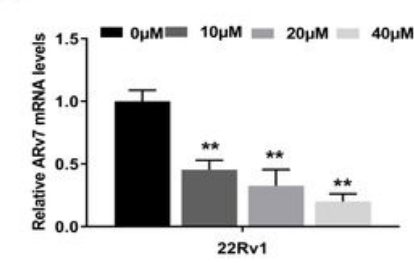

B

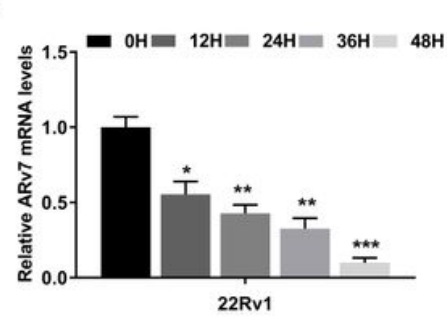

D
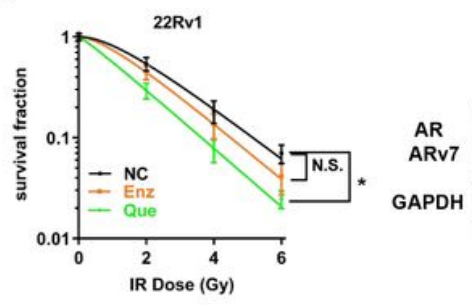

G

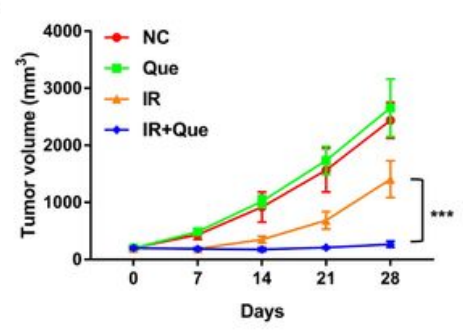

C

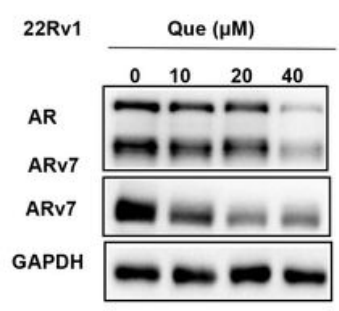

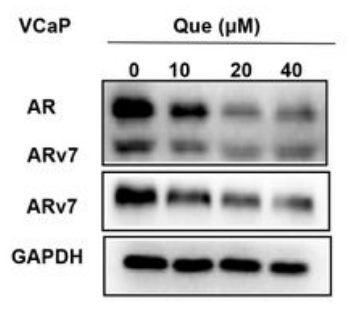

E

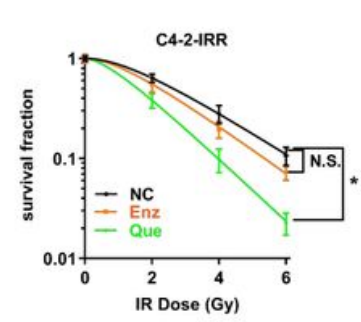

$\mathbf{F}$

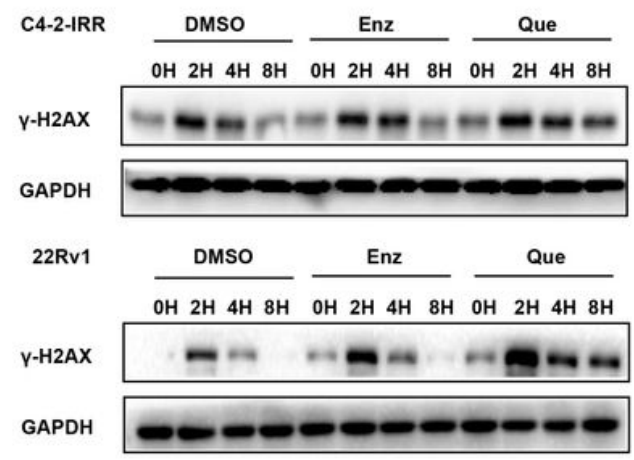

Figure 5

(A) Changes in ARv7 mRNA levels after different quercetin dose treatment. Control group is the $0 \mu \mathrm{M}$ group. (B) Changes in ARv7 mRNA after $40 \mu \mathrm{M}$ quercetin treatment at different times. Control group is the $\mathrm{OH}$ group. (C) Changes in AR and ARv7 protein levels in 22Rv1 and VCaP cells after different quercetin dose treatment. (D) Que and Enz effects on 22Rv1 cells survival after IR using clonogenic assay. (E) Que and Enz effects on C4-2-IRR cells survival after IR using clonogenic assay. (F) Western Blot analysis of $Y^{-}$ H2AX levels in DMSO, Enz and Que groups after 4Gy IR. (G) The average tumor volumes of the in vivo mouse model (each group $\mathrm{N}=6$ ) studies were recorded every 7 days and tumor volume $(\mathrm{mm} 3)$ changes were drawn. Representative Images of tumors are presented after mice were sacrificed on day 28 and the tumor volume in each group was measured. Data are presented as mean $\pm S E M$. ${ }^{*} P<0.05,{ }^{\star *} P<0.01$, $\star \star \star P<0.001$ compared with the controls. N.S., not significant compared with the controls.

\section{Supplementary Files}

This is a list of supplementary files associated with this preprint. Click to download.

- sfig1.jpg 